\title{
APOPLEXIA SUBCLÍNICA EM TUMORES PITUITÁRIOS
}

\author{
MARCELO M. PINHEIRO**, ARTHUR CUKIERT*, LUIS R. SALGADO**, \\ MARCIA NERY**, JAYME GOLDMAN**, FERNANDO PIMENTEL **, BERNARDO LIBERMAN**
}

RESUMO - Apoplexia pituitária aguda é fenômeno relativamente raro, mesmo em macroadenomas. No entanto, a presença de áreas necro-hemorrágicas intratumorais nestes tumores que não se relacionam a qualquer sintomatologia aguda parece ser bem mais frequente do que se imaginava na era da tomografia. Com o advento da ressonância magnética estas áreas podem ser facilmente diagnosticadas pré-operatoriamente. Dentre os 40 últimos macroadenomas operados e examinados com RMN, 10 possuíam áreas apopléticas subclínicas em seu interior. Sete destes tumores eram não secretores, 2 secretores de GH e um de prolactina. O quadro clínico incluiu, além daqueles correspondentes à eventual secreção endócrina, perda visual progressiva (sem história de piora abrupta ou rápida) $(n=8)$ e cefaléia $(\mathrm{n}=3)$. Após a remoção cirúrgica destes tumores e descompressão do aparato óptico, obtivemos melhora visual em 6 pacientes e em 2 a visão permaneceu inalterada. A cefaléia desapareceu em 2 casos. A presença de áreas apopléticas nestes macrotumores bem como sua ausência em séries de microtumores relatadas na literatura sugere que se relacionam mais ao tamanho do tumor do que ao seu caráter secretor ou não, o que é compatível com a provável natureza (insuficiência vascular) da apoplexia subclínica nesses casos.

PALAVRAS-CHAVE: apoplexia, tumores, pituitária, assintomáticos.

\section{Asymptomatic apoplexy in pituitary tumors}

ABSTRACT - Acute pituitary apoplexy is a rare event, even in patients with pituitary macroadenomas. On the other hand, the presence of necrotic/hemorrhagic areas, especially in macroadenomas, seems to be more common than earlier reported in the CT period. After the introduction of MR in the presurgical workup of these patients, these apopleptic areas have been more easily diagnosed preoperatively. Forty consecutive patients with pituitary macroadenomas were studied with high-resolution $1.5 \mathrm{~T}$ T1 coronal, sagittal and axial slices over the sellar region. Special attention was paid in the detection of necrotic, cystic and hemorrhagic areas within these tumors. Ten patients had hemorrhagic/necrotic areas within their tumors, without any sign or symptom of acute apoplexy. These areas varied from small $(2 \mathrm{~mm})$ to very large $(30 \mathrm{~mm})$ ones. Seven patients had non-secreting tumors, 2 GH and 1 prolactin secreting tumors, which is the same profile of secretory pattern for the whole series (40 patients). The clinical picture included (other than that caused by endocrine secretion) slowly progressive (but not acute) visual loss $(\mathrm{n}=8)$ and headache $(\mathrm{n}=3)$. After surgical decompression of the surrounding structures and visual apparatus, which was facilitated by the presence of the necrotic areas, there was visual improvement in 6 patients and headache resolution in 2 . The presence of asymptomatic apopleptic areas in these macroadenomas and their absence in microadenomas as can be seen in the literature suggest that they are related more to the size of the tumor than to its endocrine secretion pattern. This is in agreement with a vascular insufficiency hypothesis in the pathogenesis of these lesions.

KEY WORDS: apoplexy, tumors, pituitary, asymptomatic.

A apoplexia pituitária aguda pode caracterizar-se clinicamente por diminuição súbita da visão, paralisias oculomotoras, cefaléia severa, diminuição do nível de consciência, hipopituitarismo e sinais de irritação meníngea ${ }^{1-3}$. Isto geralmente ocorre em decorrência da expansão abrupta do conteúdo selar como resultado de hemorragia ou infarto de um adenoma pituitário ou, mais raramente,

Serviços de Neurocirurgia* e Endocrinologia** do Hospital Brigadeiro, São Paulo SP. Aceite: 4novembro-1998.

Dr. Arthur Cukiert - R. Nova York 744 / 131 - 04560-001 São Paulo SP - Brasil. 
em tumores não adenomatosos e em glândulas normais ${ }^{4}$. A apoplexia pituitária aguda é evento relativamente raro, ocorrendo em menos de $10 \%$ dos adenomas pituitários ${ }^{1}$. Entretanto, a presença de formação cística e hemorragia clinicamente não suspeitada pode ocorrer em mais de $25 \%$ dos casos submetidos a revisões patológicas ${ }^{5-7}$. Esses eventos silenciosos podem causar sintomas neurológicos atípicos ou podem ser clinicamente assintomáticos; esta entidade tem sido designada de apoplexia pituitária subclínica ${ }^{8,9}$. Tanto os adenomas não funcionantes como os endocrinologicamente ativos têm demonstrado alterações apopléticas, e em uma revisão geral da literatura nenhum tipo específico de tumor estava sobre maior risco; no entanto, quanto maior o tumor, maior parece ser a prevalência de áreas necróticas em seu interior ${ }^{10}$.

Na época pré-ressonância magnética nuclear (RMN), radiografias de crânio, punção lombar e tomografia computadorizada (TC) eram os meios disponíveis para realizar o diagnóstico de apoplexia pituitária. Hemorragia subaguda, entretanto, dificilmente era diagnosticada por esses métodos, e somente era reconhecida no momento da intervenção cirúrgica. Mais recentemente, a RNM tem facilitado o diagnóstico pré-operatório da apoplexia pituitária subclínica, possibilitando maior acurácia na investigação e tratamento dessas lesões ${ }^{1}$.

O presente estudo relata a experiência no diagnóstico pré-operatório de apoplexia subclínica em pacientes portadores de macrotumores pituitários através do uso de RMN.

\section{MÉTODOS}

Quarenta pacientes portadores de macroadenomas operados foram investigados com RMN de alta resolução pré-operatoriamente. Cortes finos coronais, axiais e sagitais envolvendo a região selar, com sequências em T1 com e sem contraste foram obtidos em todos os casos. Atenção especial foi dada à detecção de áreas hemorrágicas, necróticas e císticas dentro destes tumores.

A idade variou de 14 a 57 anos (26 mulheres e 14 homens). Não havia história de quadro ictal em nenhum paciente. Trinta pacientes possuíam tumores não-funcionantes, 8 tumores secretores de $\mathrm{GH}$ e 2 secretores de prolactina. Vinte e seis pacientes com tumores não funcionantes apresentavam déficit campimétrico progressivo (não agudo) e 4 cefaléia como apresentação clínica. Além dos sintomas relacionados à secreção de GH, 4 acromegálicos possuíam deficits campimétricos progressivos e 1 cefaléia. Os pacientes com prolactinoma não possuíam cefaléia ou déficit visual. Todos os pacientes foram operados por via transesfenoidal.

\section{RESULTADOS}

Dentre os 40 macroadenomas operados e examinados com RMN, 10 possuíam áreas apopléticas subclínicas em seu interior. Estas eram caracterizadas por regiões de hipossinal em T1 e hipersinal em T2 (denotando áreas de necrose pregressa) ou pela presença de áreas com componentes hemorrágicos em diversas fases de evolução, bem como por padrão reticulado de captação de contraste (Fig 1).

Áreas necróticas pequenas $(2 \mathrm{~mm})$ ou extensas $(30 \mathrm{~mm})$ puderam ser visibilizadas. Não foram visibilizadas áreas sugestivas de infarto tumoral agudo de padrão isquêmico. Sete destes tumores eram não secretores, 2 secretores de GH e um de prolactina.

O quadro clínico incluiu, além daqueles correspondentes à eventual secreção endócrina, perda visual progressiva (sem história de piora abrupta ou rápida) (n=8; 7 tumores não funcionantes, 1 tumor secretor de $\mathrm{GH}$ ) e cefaléia ( $\mathrm{n}=3 ; 2$ tumores não-secretores, 1 tumor secretor de $\mathrm{GH}$ ). Após a remoção cirúrgica destes tumores e descompressão do aparato óptico, obtivemos melhora visual em 6 pacientes ( 5 tumores não funcionantes, 1 tumor secretor de $\mathrm{GH}$ ) e em 2 a visão permaneceu inalterada. A cefaléia desapareceu em 2 casos (2 tumores não-funcionantes).

\section{DISCUSSÃO}

Neste estudo encontramos prevalência de $25 \%$ de áreas apopléticas subclínicas em macroadenomas hipofisários examinados por RMN. Isto está em concordância com outras 


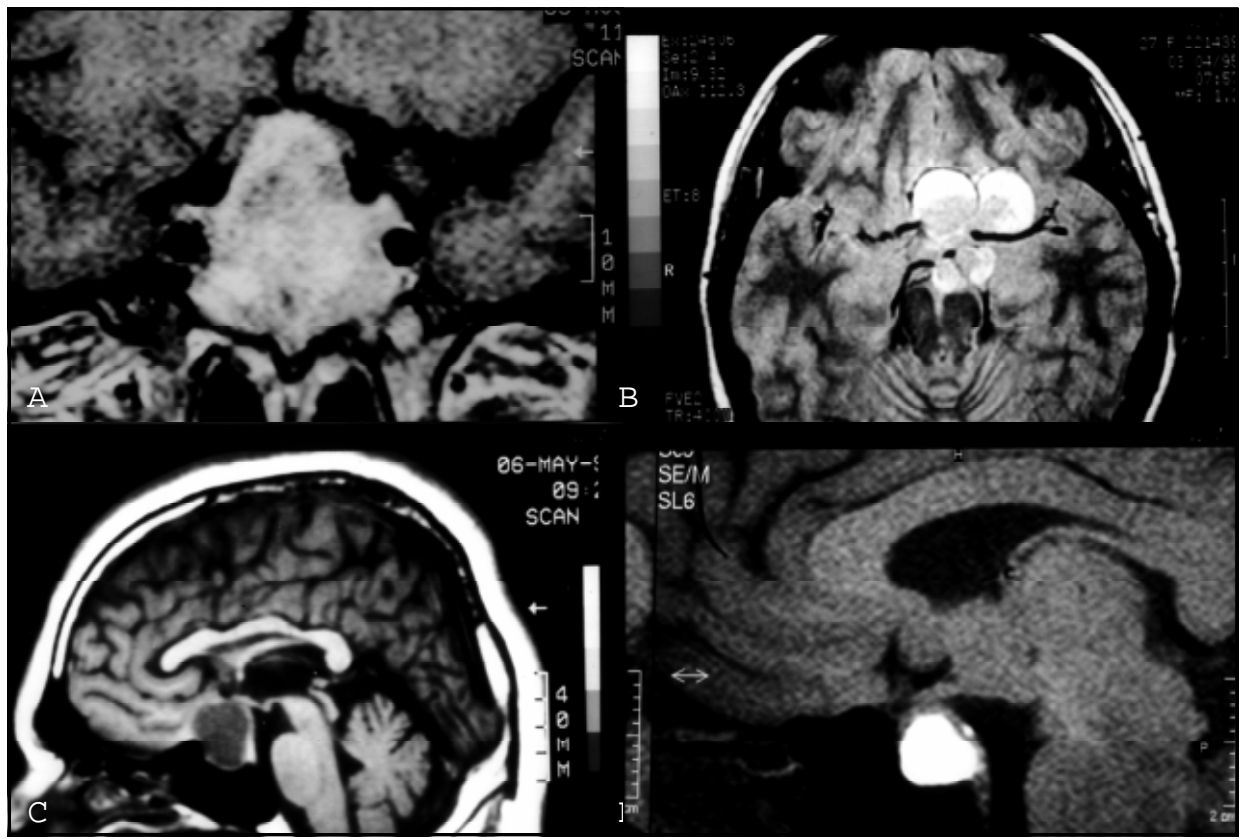

Fig 1. Imagens de apoplexia pituitária sem repercussão clínica. A: Corte sagital de RMN em T1, mostrando sangramento ocupando toda a topografia da sela; B: Corte sagital de RMN em T1 mostrando grande cisto com conteúdo hemorrágico ocupando quase todas as dimensões de macroadenoma; C: Corte axial de RMN em T2 mostrando cistos com conteúdo hemático em macroadenoma secretor de prolactina; D: Corte coronário de RMN em T1 mostrando macroadenoma com extensas áreas apopléticas em diversas fases, com características hipo-, iso- ou hiperintensas.

publicações, em que as hemorragias intra-adenomatosas em sua maioria são silenciosas ${ }^{2,11}$. Setenta e cinco por cento dos tumores hipofisários, em nossa casuística, eram não funcionantes. Crompton ${ }^{12}$ sugeriu que os tumores cromófobos conteriam vasos sanguíneos extremamente finos, quase sempre do tipo sinusoidal. Esses vasos seriam frágeis, e em alguns casos, vasos anormais poderiam ser vistos com necrose do tipo fibrinóide e hialina. Anormalidades na anatomia da parede de vasos com trombose também têm sido apontadas como causa de hemorragias em outros tumores tais como neuroma do acústico e meningiomas ${ }^{13}$. Em outra série de apoplexias ${ }^{11}$ houve número desproporcional de macroadenomas e é possível que o rápido crescimento do tumor, superando o crescimento do seu suprimento sanguíneo, seja um fator na produção de hemorragia. Onesti e col. ${ }^{2}$ salientaram que em pacientes com apoplexia subclínica não havia necrose. Em comparação, 13 de 16 pacientes com apoplexia aguda apresentavam necrose. A apoplexia clínica parecia estar relacionada ao infarto hemorrágico dentro de um tumor hipofisário; já a apoplexia subclínica resultaria de hemorragia espontânea dentro de uma neoplasia vascularizada. Na presença de infarto, o tecido necrótico pode de algum modo aumentar a probabilidade da sintomatologia apoplética.

A RMN é um método de alta resolução na detecção de hemorragia intra-selar nos estágios subagudo e crônico, enquanto a TC é mais valiosa na fase aguda da hemorragia hipofisária, principalmente nas primeiras $24-48$ horas $^{3}$. Uma vantagem adicional da RMN é que ela oferece uma estimativa da idade e tempo de curso da hemorragia ${ }^{1}$. Hemorragias com menos de 7 dias de duração (fase aguda) têm características de sinal hipointenso (ou isointenso) quando comparadas com o cérebro nas imagens em T1 e T2. Entre 7-14 dias (fase subaguda) um aumento na intensidade do sinal pode ser notado na periferia do hematoma, o centro do hematoma permanecendo hipointenso. 
O aumento da intensidade do sinal em todo o hematoma em T1 e T2 ocorre após 14 dias (fase crônica). $\mathrm{O}$ aumento do sinal dentro do hematoma pode ser notado até 1 ano após a hemorragia ${ }^{14}$. $\mathrm{O}$ aumento na intensidade do sinal dentro do hematoma é causado pela digestão da hemoglobina e formação de meta-hemoglobina a partir da (de)oxi-hemoglobina ${ }^{14-17}$.

Apoplexia pituitária aguda, embora incomum, é usualmente considerada uma emergência neurocirúrgica. Rápida reposição hormonal e cirurgia são quase sempre necessárias como procedimentos salvadores da visão e da vida do paciente ${ }^{10}$. Cirurgia para apoplexia hipofisária subaguda pode também ser necessária para melhora dos sintomas. Neste estudo, 6 dos 8 pacientes (75\%) apresentaram melhora visual após descompressão do aparato óptico, e os 2 restantes (25\%) permaneceram com visão inalterada. Dos 3 pacientes com cefaléia 2 melhoraram após o procedimento cirúrgico. Esses resultados estão em concordância com os resultados de Onesti e col. ${ }^{2}$, em que 13 de 16 pacientes $(81.2 \%)$ apresentaram melhora neurológica após tratamento neurocirúrgico. $\mathrm{O}$ grau de melhora visual parece relacionar-se mais ao grau de acometimento campimétrico pré-operatório e ao grau de palidez papilar, do que ao grau de acometimento visual isoladamente ${ }^{2}$, como ocorre nos casos de apoplexia aguda (observação pessoal).

A RMN aumentou em muito nossa capacidade diagnóstica em relação às apoplexias pituitárias subclínicas. Este diagnóstico é hoje estabelecido pré-operatoriamente e confirmado no momento da abordagem cirúrgica.

\section{REFERÊNCIAS}

1. Glick RP, Tiesi JÁ. Subacute pituitary apoplexy: clinical and magnetic resonance imaging characteristics. Neurosurgery 1990;27:214-219.

2. Onesti ST, Wisniewski T, Post KD. Clinical versus subclinical pituitary apoplexy: presentation, surgical management, and outcome in 21 patients. Neurosurgery 1990;26:980-986.

3. Ostrov SG, Quencer RM, Hoffman JC, Davis PC, Hasso AN, David NJ. Hemorrhage within pituitary adenomas: how often associated with pituitary apoplexy syndrome? AJR 1989;153:153-160.

4. Reid RL, Quigley ME, Yen SSC. Pituitary apoplexy. a review. Arch Neurol 1985;42:712-719.

5. Dolinskas CA, Bilaniuk LT, Zimmerman RA, Kul DE. Computed tomography of intracerebral hematomas: transmission CT obstructions on hematoma resolution. AJR 1977;129:601-688.

6. Fitz-Patrick D, Tolis G, McGarry EE, Taylor S. Pituitary apoplexy: the importance of skull roentgenograms and computerized tomography in diagnosis. JAMA 1980;244:59-61.

7. Mohanty S, Tandon PN, Banerju AK, Prakash B. Haemorrhage into pituitary adenomas. J Neurol Neurosurg Psychiatry 1977;40:987-991.

8. Findling JW, Tyrrell JB, Aron DC, Fitzgerald PA, Wilson CB, Forsham PH. Silent pituitary apoplexy: subclinical infarction of an adrenocorticotropin-producing pituitary adenoma. J Clin Endocrinol Metab 1981;52:95-97.

9. Peter AS. Subclinical pituitary apoplexy. NY State J Med 1986;86:656-657.

10. Cardoso ER, Peterson EW. Pituitary apoplexy: a review. Neurosurgery 1984;14:363-373.

11. Symon L, Mohanty S. Haemorrhage in pituitary tumours. Acta Neurochirurg 1982;65:41-49.

12. Crompton MR. The pathology of pituitary tumours. In Jenkins JS (ed). Pituitary tumours. London: Butterworth, 1973.

13. Castillo R, Watts C, Pullman M. Sudden haemorrhage in an acoustic neurinoma. J Neurosurg 1982;56:417-419.

14. Gomori JM, Grossman RI, Goldberg HI, Zimmerman RA, Bilaniuk LT. Intracranial hematomas: imaging by high-field MR. Radiology 1985;157:87-93.

15. Bradley WG, Schmidt PG. Effect of methemoglobin formation on the MR appearance of subarachnoid hemorrhage. Radiology 1985;156:99-103.

16. DeLaPay RL, New PFJ, Buonanno FS, et al.. NMR imaging of intracranial hemorrhage. J Comput Assist Tomogr 1984;8:599-607.

17. Kyle CA, Laster RA, Burton EM, Sanford RA. Subacute pituitary apoplexy: MR and CT appearance. J Comput Assist Tomogr 1990;14:40-44. 